\title{
A chain-type diamine strategy towards strongly anisotropic triiodide of DMEDA $I_{6}$
}

\author{
Li Yao ${ }^{1}$, Peng $\mathrm{Xu}^{2 *}$, Wanru Gao ${ }^{1}$, Junze $\mathrm{Li}^{3}$, Liang Gao ${ }^{1}$, Guangda Niu ${ }^{1}$, Dehui $\mathrm{Li}^{3}$, \\ Shiyou Chen ${ }^{4,5^{*}}$ and Jiang Tang ${ }^{1,3^{*}}$
}

\begin{abstract}
Linearly bonded triiodide chains with fairly small distance between the adjacent iodine ions feature a facile electron transfer and highly anisotropic properties. Here, we demonstrate a novel strategy towards a new one-dimensional linear triiodide DMEDA. $\mathrm{I}_{6}$, using chain-type $N, N^{\prime}$-dimethylethanediamine (DMEDA) cation to coordinate triiodine ions. This triiodide has the shortest distance between adjacent $\mathrm{I}_{3}{ }^{-}$ and good linearity. An estimated electronic band gap of $1.36 \mathrm{eV}$ indicates its semiconducting properties. 100 fold differences both in polarization-sensitive absorption and effective mass were achieved by simulation, with directions parallel and perpendicular to the $a$-axis of DMEDA.I6. The DMEDA.I6 single crystal-based photodetectors show a good switching characteristic and a distinct polarization-sensitive photoresponse with linear dichroic photodetection ratio of about 1.9. Strongly anisotropic features and semiconducting properties of DMEDA. $\mathrm{I}_{6}$ make this triiodide system an interesting candidate for polarization related applications.
\end{abstract}

Keywords: triiodide semiconductor, polarization-sensitive detection, linear dichroism

\section{INTRODUCTION}

Organic metal halide perovskites have drawn much attention in high-performance optoelectronic devices, because of their large absorption coefficient, high defect tolerance, and easy solution processing [1]. These favourable electronic properties are also found in metal-free organic halides, especially in organic polyiodide [2]. Polyiodides with various iodine skeleton dimensionalities and stoichiometries ranging from $\mathrm{I}_{2}{ }^{-}$to $\mathrm{I}_{29}{ }^{-}$[3] can be obtained by changing the surrounding counterions or organic molecule matrices. Polyiodides have attracted great attention in various application fields because of their fascinating chemical and structural diversity. For instance, the best-studied photochemical system for fundamental research, such as solid-state reactions in condensed-phase chemistry was based on the polyiodide [4]. The others were mainly used as electrolytes and redox mediators for dye-sensitized solar cells or lithium-iodide batteries $[5,6]$, or as iodine species for perovskite photovoltaics $[7,8]$. Among these polyiodides, the most studied are the triiodides, and more than 500 triiodide structures have been reported [2].

Triiodide ions are commonly stacked in T-shape or zigzag patterns, and a few special triiodides preserve infinite linear $\mathrm{I}_{3}{ }^{-}$channels [2,9]. Recently, researchers have adopted carbon nanotubes [10] or metal-organic frameworks [11] to encapsulate iodine chains, which provide perfect systems for investigating the electron dynamics and couplings along the linear iodine chain. In linear $\mathrm{I}_{3}{ }^{-}$ chain, the electron-exchange effects are largely influenced by the orbital overlap between the nearest $\mathrm{I}_{3}{ }^{-}$ions [12], and their dichroism is determined by the linear arrangement along the $\mathrm{I}_{3}{ }^{-}$ion orientation [13]. In previous studies about linear triiodides, such as tetra- $n$-butyl-

\footnotetext{
${ }^{1}$ Wuhan National Laboratory for Optoelectronics and School of Optical and Electronic Information, Huazhong University of Science and Technology, Wuhan 430074, China

${ }^{2}$ Research Institute for Magnetoelectronics \& Weak Magnetic-field Detection, College of Science, China Three Gorges University, Yichang 443002, China

${ }^{3}$ School of Optical and Electronic Information, Huazhong University of Science and Technology, Wuhan 430074, China

${ }^{4}$ State Key Laboratory of Precision Spectroscopy, Key Laboratory of Polar Materials and Devices (MOE), and Department of Optoelectronics, East China Normal University, Shanghai 200241, China

${ }^{5}$ Collaborative Innovation Center of Extreme Optics, Shanxi University, Taiyuan 030006, China

* Corresponding authors (emails: xupeng@ctgu.edu.cn (Xu P); jtang@mail.hust.edu.cn (Tang J); chensy@ee.ecnu.edu.cn (Chen S))
} 
ammonium triiodide, (benzamide) $)_{2} \cdot \mathrm{HI}_{3}$, and caffeine. $\mathrm{H}_{2} \mathrm{O} \cdot \mathrm{HI}_{3}$ [9], (dibenzo-18-crown-6) $\cdot \mathrm{H}_{2} \mathrm{O} \cdot \mathrm{HI}_{3}$ [3], mostly large cyclic organic molecules or monoamine cations were used as coordinated matrices. In these triiodides, the $\mathrm{I}_{3}^{-} \cdots \mathrm{I}_{3}^{-}$interactions were weak (typically $d\left(\mathrm{I}_{3}^{-} \cdots \mathrm{I}_{3}{ }^{-}\right) \geq$ $3.5 \AA$ ) and the arrangement of triiodide ions was largely disordered [2]. In order to improve their linear dichroism and carrier transport properties, a new design concept for linear triiodides is urgently needed.

Here, we use chain-type diamine with suitable length to coordinate $\mathrm{I}_{3}^{-}$and to produce a chain of triiodide DMEDA.I ${ }_{6}$. N,N'-dimethylethanediamine (DMEDA) cation $\left(\mathrm{CH}_{3} \mathrm{NH}_{2} \mathrm{CH}_{2} \mathrm{CH}_{2} \mathrm{NH}_{2} \mathrm{CH}_{3}{ }^{2+}\right)$ was employed in this study to stabilize the triiodide ions by hydrogen-bond interaction $(\mathrm{N}-\mathrm{H} \cdots \mathrm{I})$. Strong absorption anisotropy along the directions parallel and perpendicular to the I-I-I chain axis was confirmed experimentally and theoretically. We also explored the photoresponse properties of this triiodide single crystal for polarization-sensitive photodetection (dichroic ratio $\sim 1.9$ ), and this unoptimized performance was comparable to some single crystalline perovskite nanowires [14] or two-dimensional (2D) GeAs [15].

\section{METHODS}

\section{Sample fabrication}

DMEDA $(\geq 90 \%)$ was purchased from Alfa Aesar. HI acid, hexane and $\mathrm{I}_{2}$ pills were bought from Sinopharm Chemical Reagent Co., Ltd., China. N,N'-dimethylformamide (DMF, $\geq 95 \%$ ) was purchased from Aladdin. All materials and reagents were used as received. For the preparation of DMEDA. $\mathrm{I}_{6}$ single crystals, $5 \mathrm{mmol}$ DMEDA and $5 \mathrm{mmol} \mathrm{I}_{2}$ powder were put into $10 \mathrm{~mL}$ of $\mathrm{HI}$ acid. Then the temperature was raised to about $120^{\circ} \mathrm{C}$ and kept at this temperature until all $\mathrm{I}_{2}$ powder was fully dissolved. Afterwards, the temperature was slowly decreased to initiate crystal growth producing $3 \times 1.5 \times 1 \mathrm{~mm}^{3}$ size DMEDA $\mathrm{I}_{6}$ single crystals. These single crystals were washed with hexane and dried naturally in the air for further characterization. For the preparation of DMEDA $\cdot I_{6}$ single crystal wafers, the DMEDA $\cdot I_{6}$ single crystals were dissolved in DMF with a concentration of $2 \mathrm{~mol} \mathrm{~L}^{-1}$. After the drop of $50 \mu \mathrm{L}$ DMEDA $\cdot \mathrm{I}_{6}$ solution between two glass sheets, it was put in oven heated to $100^{\circ} \mathrm{C}$ to evaporate the solvent and start the growth of the single crystal. The final crystal phase was confirmed by the Raman spectroscopy. The DMEDA. I $_{6}$ single crystal wafers prepared by the aforementioned method had some blemishes and cracks, so they were not suitable for pho- todetector application.

\section{Material and device characterization}

$\mathrm{X}$-ray diffraction (XRD) data of the single crystals were collected using an XtaLAB PRO MM007HF diffractometer at room temperature with $\mathrm{Cu} \mathrm{Ka}$ radiation. The structure determination and refinement were conducted using Olex2. For the images presented in the manuscript, we used VESTA to visualize the crystal structure. Powder XRD (PXPD) measurements were performed by Philips X'pert PRO MRD diffractometer with $\mathrm{Cu} \mathrm{Ka}$ radiation. Thermal gravimetric analysis (TGA) was measured by a PerkinElmer Diamond TG/ DTA6300 system with a heating rate of $10^{\circ} \mathrm{C} \mathrm{min}^{-1}$ from room temperature to $600^{\circ} \mathrm{C}$ in $\mathrm{N}_{2}$ flow. The absorption spectra were measured by a UV-vis spectrophotometer (PerkinElmer Instruments, Lambda 950 using integrating sphere). The polarized absorbance spectra were measured by a MSV-5200 microscopic spectrophotometer (JASCO) in absorbance mode. The diameter of in and out circular apertures were both $100 \mu \mathrm{m}$. The polarized reflection spectra were measured in a back scattering geometry with 50X achromatic objective lens. In collecting light path, a half-waveplate (HWP) followed with a polarizer was used to get the reflected light intensity in different polarized directions through rotating the HWP, and finally, the light was collected by the Horiba iHR550 imaging spectrometer equipped with a $600 \mathrm{gr} \mathrm{mm}^{-1}$ grating. A depolarized halogen tungsten white light was used as light source. A silver mirror (Daheng Optics, GCC-10220) was used as reference sample to get the calibrated reflection light spectra. For the polarization-Raman measurements, a rotating HWP was placed in the incident light path of the Raman system (Horiba) to get the polarized light in different directions. The excitation laser was $532 \mathrm{~nm}$. To avoid laser-induced sample damage, Raman spectra were recorded at low power levels $\left(\sim 2 \mu \mathrm{W} \mu \mathrm{m}^{-2}\right)$.

For fabrication of the DMEDA. $\mathrm{I}_{6}$ photodetectors, $\mathrm{Au}$ electrodes were deposited through a mask by the thermal evaporation. Photoresponse characterizations were carried out using an Agilent parameter analyser B1500A. The channels of the single crystal devices were $10 \mu \mathrm{m}$. The 365, 395, 430, 490, 530, 590, 780, and $970 \mathrm{~nm}$ LEDs (Thorlabs) were used as the light source.

The polarization-sensitive photodetection system with $590 \mathrm{~nm}$ LED (Thorlabs M590L3), and a polarizer (Thorlabs, LPVIS050, 550-1550 nm) were used. All photo detection device performance characterizations were done in an optically and electrically sealed box to minimize the electromagnetic disturbance. 


\section{Theoretical calculation}

All the first-principles calculations were performed within the framework of the density functional theory (DFT) as implemented in VASP. The projector augmented-wave potentials were employed with an energy cutoff of $400 \mathrm{eV}$ for the plane-wave basis set. We used the Perdew Burke Ernzerhof (PBE) form of the generalized gradient approximation to the exchange-correlation potential. All the atomic coordinates were fully relaxed until the force on each atom was smaller than $0.01 \mathrm{eV} \AA^{-1}$. A $2 \times 2 \times 2 k$-mesh in the Brillouin zone was used for the $1 \times 2 \times 1$ supercell in calculating the defect properties and the equivalent $\mathrm{k}$-meshes were used for all other calculations. To predict the accurate band gap, the total energy and band structure were also calculated by the hybrid functional with a mixing parameter of $25 \%$ (HSE06).

\section{RESULTS AND DISCUSSION}

We began our study by choosing DMEDA to synthesize polyiodide (details are shown in Supplementary information). This chain-type DMEDA was selected for its unique $\mathrm{N}$... N distance $(3.38 \AA$ ) and its entire length $(6.25 \AA)$, which were equal to the typical I-I distance and I-I-I length, respectively. Using a simple solution-based growth method as described in the experimental section, we were able to grow large DMEDA $I_{6}$ single crystals which had a metallic luster and cyan hue (Fig. 1a).

The structures of these crystals were determined by single crystal XRD (SCXRD), and their chemical formulas were determined as DMEDA.I ${ }_{6}$. These triiodides crystallize into the monoclinic space group of $I_{2 / m}$ (12). The detailed crystalline structure information is provided in Table 1. In the $a$ axis projection (Fig. 1b), the structure of DMEDA $\cdot \mathrm{I}_{6}$ can be visualized as six $\mathrm{I}-\mathrm{I}-\mathrm{I}^{-}$chains arranged in a hexagonal shape with one DMEDA ${ }^{2+}$ chain occupying the cavity in the center of the framework. The I-I-I chains have electrostatic interactions with the surrounding DMEDA ${ }^{2+}$ chains. As shown in Fig. 1c, these $\mathrm{I}_{3}{ }^{-}$are stacked end to end, forming an infinite linear chain. The $\mathrm{I}_{3}{ }^{-}$are largely asymmetric and they have I-I distance of $\mathrm{I}(1)-\mathrm{I}(2) 2.78 \AA$ and $\mathrm{I}(2)-\mathrm{I}(3) 3.37 \AA$. The $\mathrm{I}_{3}{ }^{-} \ldots \mathrm{I}_{3}{ }^{-}$interactions here are relatively strong, with a distance of $3.42 \AA$. This distance is shorter than that in previously reported infinite triiodide ions chains (distance $\geq 3.5 \AA$ ). On the other hand, the I-I-I angle is $179.1(15)^{\circ}$ $[I(1)-I(2)-I(3)]$, and the I-I-I angles for the neighbouring $\mathrm{I}_{3}^{-}$are also more than $176.0^{\circ}$, which demonstrates that triiodide forms the most ordered straight $\mathrm{I}_{3}^{-}$chains obtained thus far [2].

For a close look, as presented in Fig. 1d, the terminated I of four $\mathrm{I}_{3}^{-}$are directly coordinated with one DMEDA ${ }^{2+}$ through hydrogen bonding with identical distances of 2.79(6) $\AA$, which is within the range of reported values for other polyiodide compounds [2]. The hydrogen bonding between the DMEDA ${ }^{2+}$ and $\mathrm{I}_{3}{ }^{-}$acts as connection to constrain the $\mathrm{I}_{3}{ }^{-}$chain straightly and closely. The crystal packing is stabilized by successive $\mathrm{N}-\mathrm{H}$-..I interactions.

We first studied the fundamental material properties of DMEDA. $I_{6}$. As shown in Fig. 2a, the PXRD pattern of the triiodide agrees well with their simulated PXRD pattern. $\mathrm{X}$-ray photoelectron spectroscopy (XPS) analysis of DMEDA $\cdot I_{6}$ single crystals was performed after argon ion

a

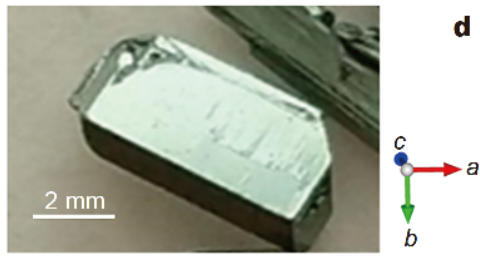

d

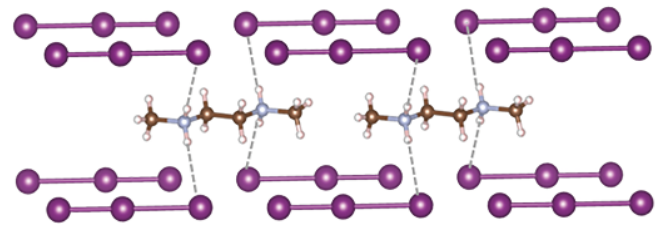

c
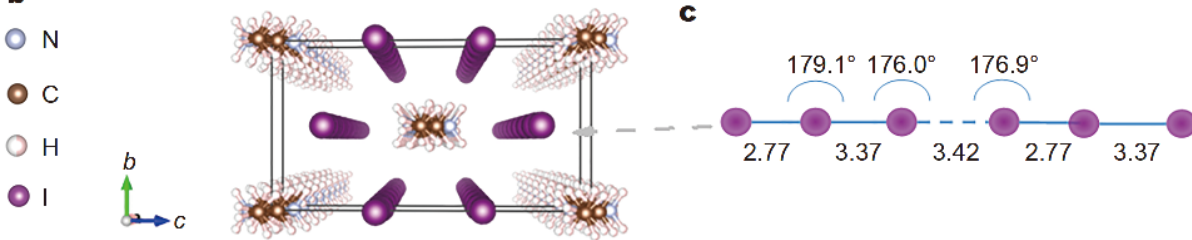

Figure 1 (a) Optical picture of DMEDA $\cdot I_{6}$ single crystal. (b) Perspective view of the crystal structure of DMEDA $I_{6}$ through the crystallographic $a$-axis projection. The volume of a unit cell is pointed out by the black parallel hexahedron. Here, I atoms are depicted as purple spheres. $\mathrm{C}, \mathrm{N}$ and $\mathrm{H}$ atoms are shown in brown, light cyan and light red, respectively. (c) The schematic structure of linear $\mathrm{I}_{3}{ }^{-}$chain in DMEDA. $\mathrm{I}_{6}$ crystal. The corresponding opening I-I-I angles and I-I bond lengths (unit: $\AA$ ) are provided. (d) Selected parts of DMEDA.I ${ }_{6}$ crystal structure for understanding the role of diamine (DMEDA). The hydrogen bonds are shown by dashed lines. 
Table 1 Crystal data and structure refinement for DMEDA $\mathrm{I}_{6}$

\begin{tabular}{|c|c|}
\hline Category & Data \\
\hline Empirical formula & $\mathrm{C}_{4} \mathrm{H}_{14} \mathrm{~N}_{2} \mathrm{I}_{6}$ \\
\hline Formula weight & 427.80 \\
\hline Temperature (K) & $299.0(5)$ \\
\hline Crystal system & monoclinic \\
\hline Space group & $I_{2 / m}$ \\
\hline$a(\AA)$ & $9.5624(19)$ \\
\hline$b(\AA)$ & $6.7653(14)$ \\
\hline$c(\AA)$ & $12.914(3)$ \\
\hline$\alpha\left(^{\circ}\right)$ & 90 \\
\hline$\beta\left({ }^{\circ}\right)$ & $100.86(3)$ \\
\hline$\gamma\left({ }^{\circ}\right)$ & 90 \\
\hline Volume $\left(\AA^{3}\right)$ & $820.5(3)$ \\
\hline$Z$ & 2 \\
\hline$\rho_{\text {calc }}\left(\mathrm{g} \mathrm{cm}^{-3}\right)$ & 3.463 \\
\hline$\mu\left(\mathrm{mm}^{-1}\right)$ & 11.329 \\
\hline$F(000)$ & 748.0 \\
\hline Crystal size $\left(\mathrm{mm}^{3}\right)$ & $0.1 \times 0.05 \times 0.02$ \\
\hline Radiation & $\mathrm{Cu} \mathrm{Ka}(\lambda=1.54184)$ \\
\hline $2 \theta$ range for data collection $\left(^{\circ}\right)$ & 4.886 to 52.59 \\
\hline Index ranges & $-11 \leq h \leq 11,-8 \leq k \leq 8,-15 \leq l \leq 16$ \\
\hline Reflections collected & 8090 \\
\hline Independent reflections & $903\left[R_{\text {int }}=0.0772, R_{\text {sigma }}=0.0280\right]$ \\
\hline Data/restraints/parameters & $903 / 0 / 38$ \\
\hline Goodness-of-fit on $F^{2}$ & 1.207 \\
\hline Final $R$ indices $[\mathrm{I}>=2 \sigma(I)]$ & $R_{1}=0.0402, w R_{2}=0.0903$ \\
\hline Final $R$ indices [all data] & $R_{1}=0.0408, w R_{2}=0.0907$ \\
\hline Largest diff. peak/hole (e $\AA^{-3}$ ) & $1.05 /-4.07$ \\
\hline
\end{tabular}

etching. The general XPS spectra (Fig. S1) confirm the primary elementary compositions of iodine, carbon, and nitrogen. In Fig. 2b, the high-resolution XPS spectrum of

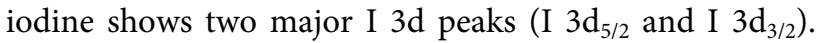
After peak separation, the $\mathrm{I} 3 \mathrm{~d}_{5 / 2}$ and $\mathrm{I} 3 \mathrm{~d}_{3 / 2}$ bands consist of two components, which may directly point to two types of I-I interactions [16]. One is from the typical $\mathrm{I}^{-}$at 618.6 and $630.1 \mathrm{eV}(76 \%)$, and the other higher binding energies are at 620.0 and $631.6 \mathrm{eV}(24 \%)$. In Fig. 2c, the optical absorption spectrum of DMEDA. $\mathrm{I}_{6}$ powder was measured by a UV-vis-NIR spectrophotometer in diffuse reflection mode. The optical bandgap of DMEDA. $\mathrm{I}_{6}$ was evaluated to be $1.36 \mathrm{eV}$ using a Tauc plot (a plot of $(A h v)^{2}$ as a function of photon energy $h v$ for a direct transition, where $A$ represents the absorbance). As shown in TGA (Fig. 2d), DMEDA. $\mathrm{I}_{6}$ is stable up to $136^{\circ} \mathrm{C}$. From the differential scanning calorimetry (DSC) analysis, there are two obvious endothermic decomposition processes. The first loss of mass of $60 \%$ comes from the evaporation of $\mathrm{I}_{2}$ (from DMEDA. $\mathrm{I}_{6}$ to DMEDA. $\mathrm{I}_{2}$ ), and the latter mainly comes from the loss of DMEDA $\cdot \mathrm{I}_{2}$.

To get a better insight into the properties of DMEDA. $\mathrm{I}_{6}$, we performed a theoretical investigation based on the DFT calculations. Fig. 3a displays the band structure and partial density of states (pDOS) calculated using the hybrid functional. The calculated band structure shows a direct bandgap of $1.26 \mathrm{eV}$ at $\mathrm{K}$ point $(0.5,0$, 0.5 ). The flat band edges indicate relatively heavy holes and electrons. The electrical conduction mechanism of DMEDA $\cdot I_{6}$ might be the successive electron transfer between $\mathrm{I}^{-}$and $\mathrm{I}_{2}$, resulting in the delocalization of electrons through the crystal. Therefore, the $\mathrm{I}^{-}$and $\mathrm{I}_{2}$ act as the donor and acceptor, respectively. Meanwhile, the DMEDA $^{2+}$ acts as an isolator. As shown in Fig. $3 \mathrm{a}$, the valence band maximum (VBM) and the conduction band minimum (CBM) are both derived from I 5p orbitals. The corresponding partial charge density plots of the VBM and the CBM are also provided in Fig. S2. Furthermore, the calculated absorption coefficient $(\alpha)$ is about $10^{6} \mathrm{~cm}^{-1}$ at around $530 \mathrm{~nm}$, which is comparable to that of $\mathrm{CH}_{3} \mathrm{NH}_{3} \mathrm{PbI}_{3}$ perovskite [1]. Note that the calculated $\alpha$ (Fig. 3b) shows an almost 100-fold difference within the entire visible range, and closely 1000 -fold difference at around $530 \mathrm{~nm}$ for two absorption directions (//a and $\perp a)$. As listed in Table 2, the calculated effective masses of holes and electrons along the $\mathrm{I}-\mathrm{I}-\mathrm{I}^{-}$chain (along the $a$ axis) are $1.69 m_{0}$ and $1.48 m_{0}$, respectively, which are about 7 times larger than those in $\mathrm{CH}_{3} \mathrm{NH}_{3} \mathrm{PbI}_{3}\left(m_{\mathrm{h}}{ }^{*}=0.29 m_{0}\right.$ and $\left.m_{\mathrm{e}}{ }^{*}=0.23 m_{0}\right)$ [17], but are comparable to that of the black phosphorene along $G-Y$ directions $\left(m_{\mathrm{h}}{ }^{*}=6.35 m_{0}\right.$ and $m_{\mathrm{e}}{ }^{*}=1.12 m_{0}$, respectively) [18]. The effective masses of holes and electrons along $b$ axis are about $185 m_{0}$ and $108 m_{0}$, and along $c$ axis are about $542 m_{0}$ and $622 m_{0}$, respectively, which indicates more than 100 -fold difference anisotropy in carrier transport characteristics.

Then we focused on the anisotropic properties of DMEDA $\cdot I_{6}$. The polarization-resolved reflection spectra were employed to explore its optical anisotropy. The measurement was conducted on the surface of single crystal using unpolarized white illumination, with collecting reflection light intensity in different polarization directions. The reflection spectrum of a silver mirror was used as a reference to calibrate the system. As shown in Fig. 4a, around $530 \mathrm{~nm}$, we can see the reflection of single crystal is $\sim 79 \%$ parallel to the $a$ axis ( along the direction of $\mathrm{I}_{-} \mathrm{I}_{-} \mathrm{I}^{-}$chain ), and is $\sim 10 \%$ in perpendicular to the $a$ 

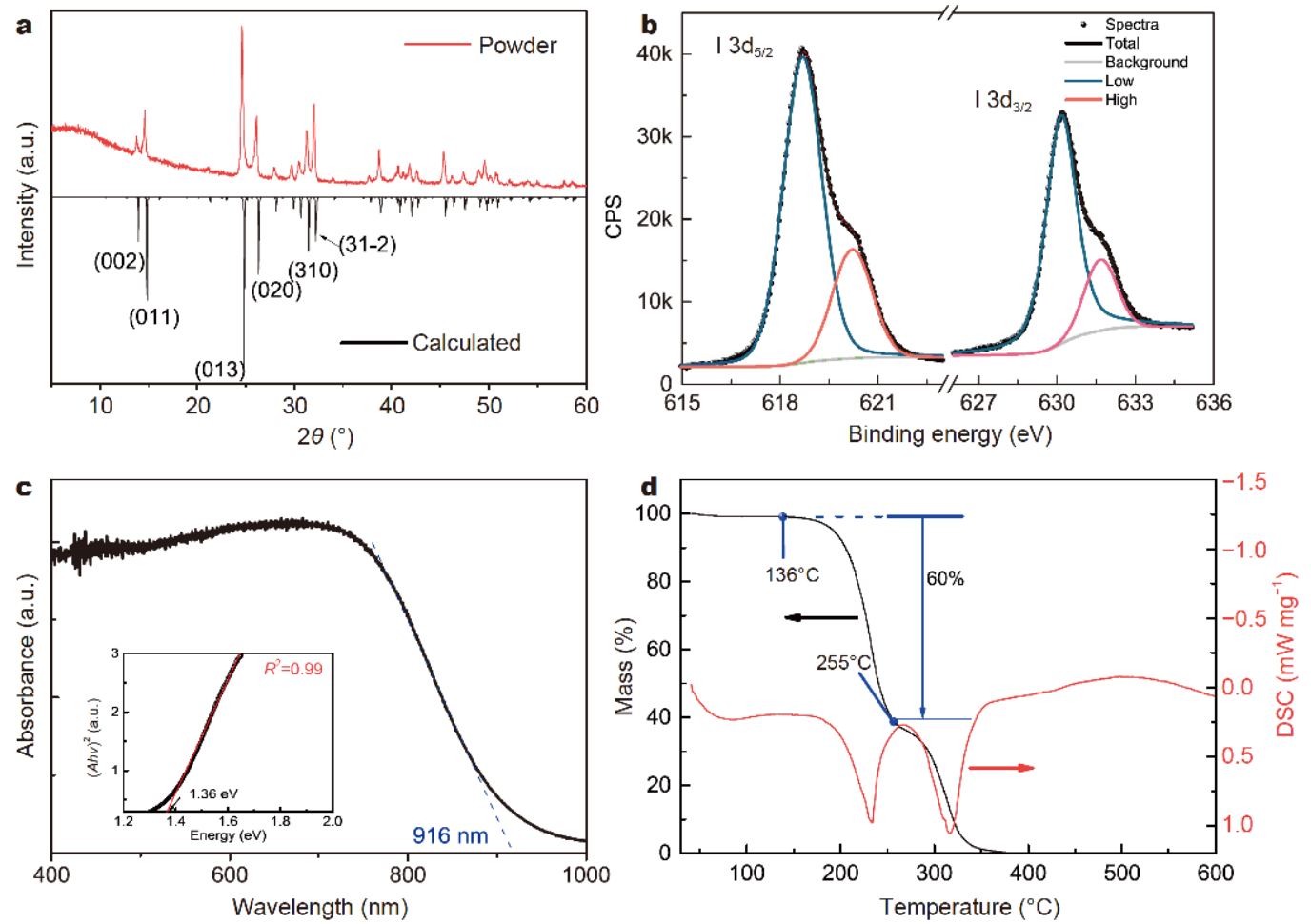

Figure 2 (a) The experimental PXRD compared with calculated XRD pattern of DMEDA.I I $_{6}$ (b) The high-resolution XPS spectra of DMEDA.I ${ }_{6}$ single crystal surface after argon ion etching. (c) The absorption spectra of the DMEDA $\cdot \mathrm{I}_{6}$ powder. The inset is the Tauc plot of DMEDA $\mathrm{I}_{6}$ for a direct bandgap semiconductor. (d) The TGA and DSC spectra of DMEDA.I $\mathrm{I}_{6}$.
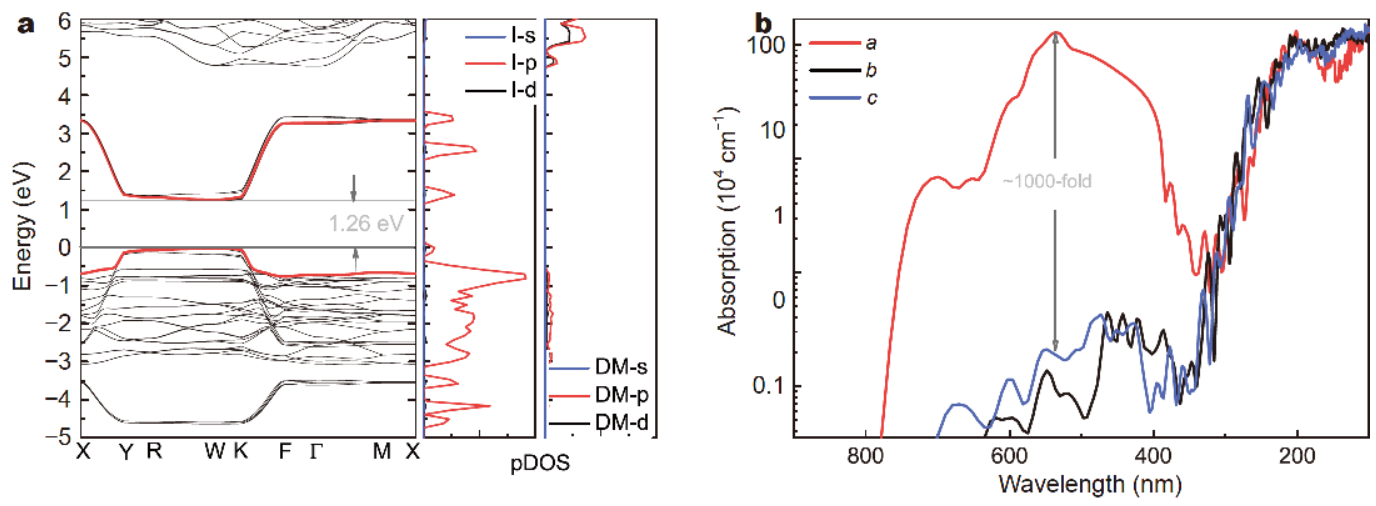

Figure 3 (a) The DFT calculated band structure (left panel) of DMEDA $\mathrm{I}_{6}$ and its partial density of states (pDOS, right panel) projected on DMEDA (marked as DM) and I. (b) Calculated absorption coefficient (by HSE06 functional) along three directions. Here, the $a$ axis represents the direction along the $\mathrm{I}-\mathrm{I}^{-} \mathrm{I}^{-}$chain.

axis. This clearly signifies the large reflective anisotropy of these polyiodide complexes.

The polarized absorbance spectrum of DMEDA. $\mathrm{I}_{6}$ was measured on a microscopic spectrophotometer in absorbance mode. The single crystal wafer used for measurement was about $12 \mu \mathrm{m}$ thick. The measurement region was within the circle (diameter: $100 \mu \mathrm{m}$ ) marked in the two insets of Fig. 4b. The two inset panels were taken from the single crystal wafer under the polarization light with directions parallel (top panel) and perpendicular (bottom panel) to the $a$ axis, respectively. Here, the 10 -fold polarization absorbance difference can reach 
Table 2 The calculated effective masses of electrons and holes, and the absorption coefficient estimated from the calculated band structures along the three directions of DMEDA $\cdot \mathrm{I}_{6}$ single crystal

\begin{tabular}{cccc}
\hline Directions & $m_{\mathrm{e}}{ }^{*} / m_{0}$ & $m_{\mathrm{h}}{ }^{*} / m_{0}$ & $\alpha\left(\times 10^{4} \mathrm{~cm}^{-1}, @ 536 \mathrm{~nm}\right)$ \\
\hline$a$ axis & 1.48 & 1.69 & 130 \\
$b$ axis & 108 & 185 & 0.09 \\
$c$ axis & 622 & 542 & 0.19 \\
\hline
\end{tabular}

around the absorption edge, which is less than the theoretical data ( 100 -fold difference).

We also used the polarized Raman spectroscopy to study the anisotropy of DMEDA $\mathrm{I}_{6}$. This polarized Raman characterization is widely used in investigating the anisotropy of 2D materials [15,18]. Besides, Raman spectroscopy can also be used to investigate the nature of the iodide species in DMEDA $\mathrm{I}_{6}$. The Raman spectra were measured on single crystal sample using the $532 \mathrm{~nm}$ excitation laser. As shown in Fig. 4c, the vibrational spectra of the triiodide ion have one asymmetric stretching band of $v_{1}$ at $160 \mathrm{~cm}^{-1}$ with the strongest intensity. The Raman resonance of 321 and $482 \mathrm{~cm}^{-1}$ are assigned to the $2 v_{1}$ and
$3 v_{1}$ vibrational mode, respectively. The low-frequency band at around $98 \mathrm{~cm}^{-1}$ can be assigned to the symmetric stretching vibration of $\mathrm{I}_{3}^{-}$[19]. As the laser polarization direction rotates with $30^{\circ}$ per step, the intensity of the four Raman peaks all change along with the polarization angle with the same sensitivity. Here, we provide the $160 \mathrm{~cm}^{-1}$ peaks at various polarization angles in polar coordinate in Fig. 4d. The anisotropy variation period is $180^{\circ}$. A 100 -fold difference in Raman intensities is shown in the polar plot under the laser polarization along and perpendicular to the $a$ axis of crystal.

The photoconductive properties of DMEDA. $\mathrm{I}_{6}$ were studied based on single crystals. As shown in Fig. 5a, at a bias of $1 \mathrm{~V}$, the dark current is as low as $5 \mathrm{pA}$. Under monochromatic $590 \mathrm{~nm}$ light illumination, the photocurrent achieves $1.4 \mathrm{nA}$ under a light power density of $17.2 \mathrm{~mW} \mathrm{~cm}^{-2}$, which gives a light-to-dark current ratio of $\sim 280$. The response of photocurrent to optical pulse was measured at $10 \mathrm{~s}$ intervals. The dynamic photo response of the triiodide device shows excellent switching characteristic for its stability on the "on" and "off" state and good ambient stability (Fig. S3). From Fig. 5b, we can
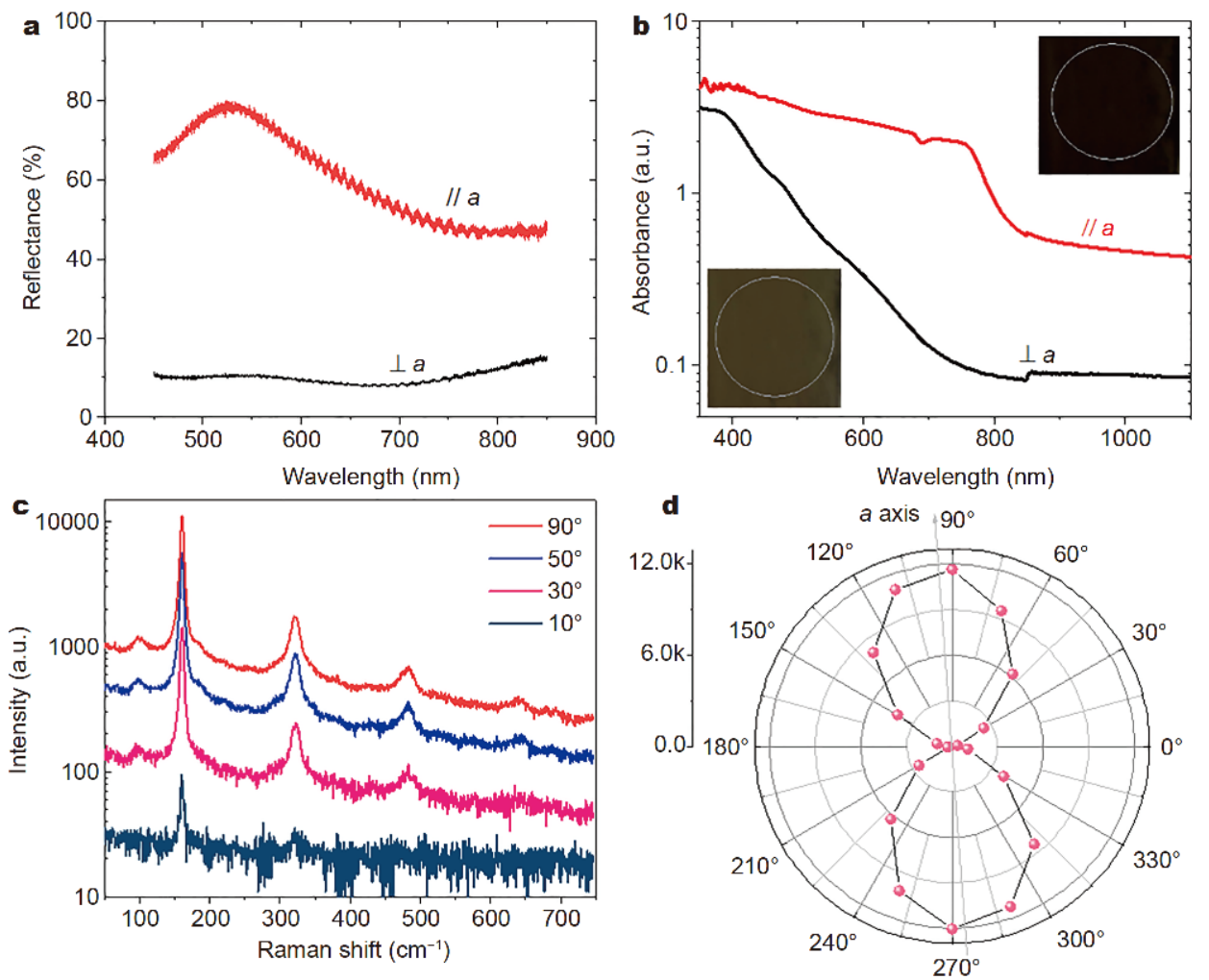

Figure 4 (a) Polarized reflection spectra of DMEDA $\cdot \mathrm{I}_{6}$ crystal along and perpendicular to the $a$ axis. (b) Polarized absorbance spectra of DMEDA $\mathrm{I}_{6}$ crystal wafer along and perpendicular to the $a$ axis. (c) Polarized Raman spectra of DMEDA $I_{6}$ crystal as different polarization angles of the excitation laser. (d) Polarization dependence of the Raman peak intensity at $160 \mathrm{~cm}^{-1}$ for DMEDA. $\mathrm{I}_{6}$ crystal in polar coordinate. 

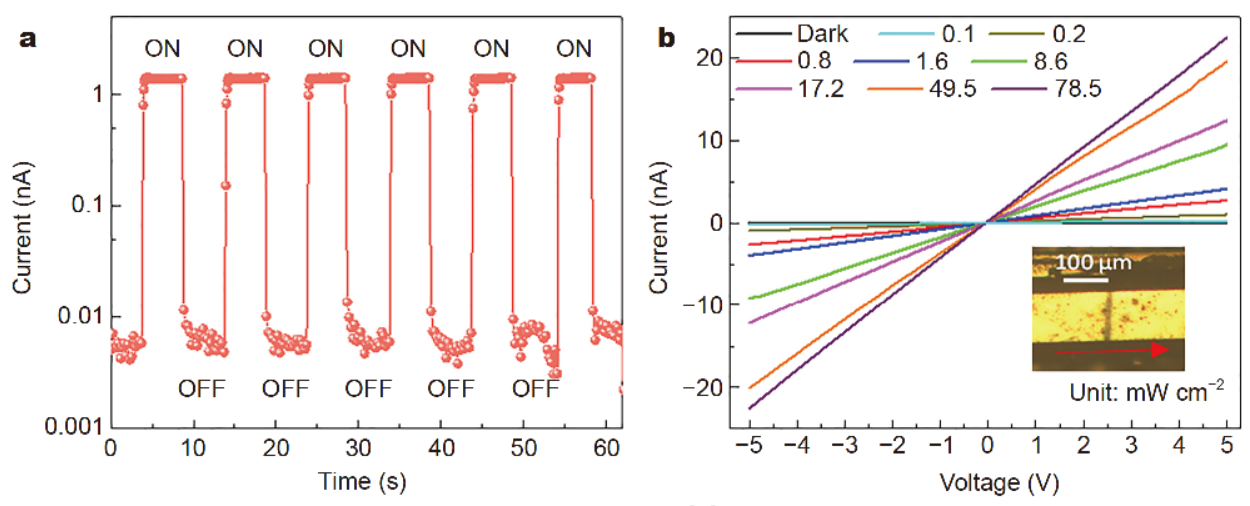

c

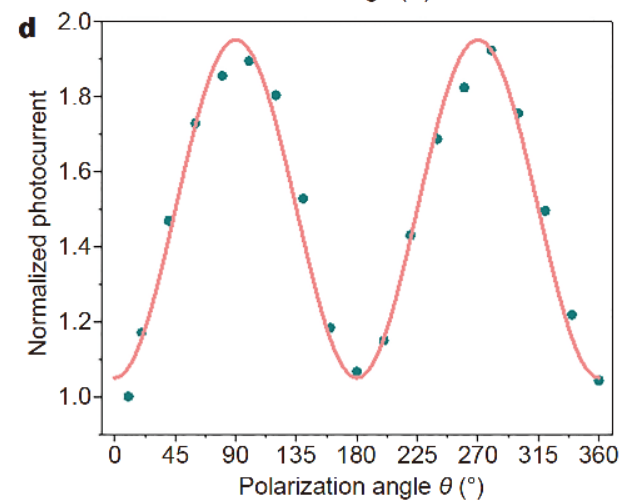

Figure 5 (a) Photoresponse characteristic of DMEDA. $\mathrm{I}_{6}$ single crystal photoconductive photodetector under $1 \mathrm{~V}$ bias and $590 \mathrm{~nm}$ monochromatic illumination with light intensity of $17.2 \mathrm{~mW} \mathrm{~cm}^{-2}$. (b) Typical $I$ - $V$ characteristics of the DMEDA $\mathrm{I}_{6}$ single crystal photodetector: dark current and photocurrent under different illumination intensities of $590 \mathrm{~nm}$. The inset is the picture of the DMEDA. $\mathrm{I}_{6}$ single crystal photoconductive photodetector. The $a$ axis direction is marked by red arrow. (c) Schematic measurement setup for anisotropic polarization sensitive photoresponse for DMEDA $\cdot I_{6}$ single crystal. (d) Experimental polarization-sensitive photocurrents for DMEDA. $I_{6}$ single crystal.

see the device shows a typical Ohmic contact under different light illumination intensities (ranging from 0.05 to $78.5 \mathrm{~mW} \mathrm{~cm}^{-2}$ of $590 \mathrm{~nm}$ light illumination). The wavelength dependent responsivity $(R)$ is calculated as: $R=\left(I_{\mathrm{p}}\right.$ $\left.-I_{\mathrm{d}}\right) /(S \times P)$, where $I_{\mathrm{p}}$ is the photocurrent, $I_{\mathrm{d}}$ is the dark current, $P$ is the light power density, $S$ is the active area of the device. The responsivity of the single crystal device is about $1 \mathrm{~A} \mathrm{~W}^{-1}$ under $10 \mu \mathrm{W} \mathrm{cm}{ }^{-2}$, and reduces to $\sim 0.01 \mathrm{~A} \mathrm{~W}^{-1}$ under $8.6 \mathrm{~mW} \mathrm{~cm}^{-2}$ for $590 \mathrm{~nm}$ illumination. The wavelength-dependent responsivity under $\sim 10 \mu \mathrm{W} \mathrm{cm}{ }^{-1}$ is provided in Fig. S4. The spectral photoresponse of our photodetector demonstrates its broadband detection capability covering the entire visible range.

As shown in Fig. 5c, the linear-polarization test system consists of a $590 \mathrm{~nm}$ LED source with depolarized illumination, and a polarizer. Before detection, the light intensity was checked to keep identical as one cycle rotation of the polarizer. The photocurrent of the DMEDA $\mathrm{I}_{6}$ single crystal device is plotted in Fig. 5d with light polarization ranging from $0^{\circ}$ to $360^{\circ}$ with intervals of $20^{\circ}$.
The linear dichroic photodetection ratio defined as $I_{\text {pmax }} /$ $I_{\text {pmin }}$ is about 1.9. Here, $I_{\text {pmax }}$ and $I_{\text {pmin }}$ are the maximum and minimum photocurrents detected by rotating the polarizer, respectively. The single crystal measured here is about $200 \mu \mathrm{m}$ thick. For a photoconductive photodetector, the photoresponse is represented by the product of photogenerated carriers and carrier mobility. In our case, the electrodes are fixed so the charge transfer always occurs along the $a$-axis, and the photoresponse anisotropy solely originates from the difference in the concentration of photogenerated carriers, which is determined by the absorption. We also measured the polarized absorption spectrum of $18-\mu \mathrm{m}$ thick single crystal wafers (Fig. S5). The polarization absorbance difference reduces from 5 -fold to about 2 -fold when the thickness increases from 12 to $18 \mu \mathrm{m}$. That means using thick single crystal $(200 \mu \mathrm{m}$ thick) to fabricate photodetector, the polarization absorbance difference may be very small. Therefore, the linear dichroic photodetection ratio of this triiodide device does not reflect the large linear dichroism of this triiodide as predicted theoreti- 
cally. We believe that more than 10 times of polarizationsensitive photocurrent can be realized in the future by fabricating optimized-thick single crystalline film.

\section{CONCLUSIONS}

In conclusion, we have demonstrated a novel triiodide semiconductor with strong absorption anisotropy, which makes it attractive for polarization-sensitive filters and photodetectors. Chain-type diamine cations $\left(\mathrm{DMEDA}^{2+}\right)$ have been used to assist the formation of infinite $\mathrm{I}_{3 n}{ }^{-}$ chains in single crystals. For the entire visible range, a 100 -fold difference in calculated absorption coefficients was obtained between two orthogonal crystallographic axis directions (parallel and perpendicular to the $a$ axis) of DMEDA. $I_{6}$. This excellent polarization-sensitive absorption was confirmed by the angle-resolved Raman spectroscopy and experimentally measured absorbance spectra. Strong anisotropic absorption combined with a good photoresponse should enable their polarization related optoelectronic applications.

Received 31 August 2019; accepted 18 October 2019; published online 20 November 2019

1 Green MA, Ho-Baillie A, Snaith HJ. The emergence of perovskite solar cells. Nat Photon, 2014, 8: 506-514

2 Svensson PH, Kloo L. Synthesis, structure, and bonding in polyiodide and metal iodide-iodine systems. Chem Rev, 2003, 103: 1649-1684

3 Kloo L, Svensson PH, Taylor MJ. Investigations of the polyiodides $\mathrm{H}_{3} \mathrm{O} \cdot \mathrm{I}_{x}(x=3,5$ or 7$)$ as dibenzo-18-crown- 6 complexes. J Chem Soc Dalton Trans, 2000, 1061-1065

4 Xian R, Corthey G, Rogers DM, et al. Coherent ultrafast latticedirected reaction dynamics of triiodide anion photodissociation. Nat Chem, 2017, 9: 516-522

5 Wood CJ, McGregor CA, Gibson EA. Does iodine or thiocyanate play a role in p-type dye-sensitized solar cells? ChemElectroChem, 2016, 3: 1827-1836

6 Boschloo G, Gibson EA, Hagfeldt A. Photomodulated voltammetry of iodide/triiodide redox electrolytes and its relevance to dyesensitized solar cells. J Phys Chem Lett, 2011, 2: 3016-3020

7 Turkevych I, Kazaoui S, Belich NA, et al. Strategic advantages of reactive polyiodide melts for scalable perovskite photovoltaics. Nat Nanotech, 2019, 14: 57-63

8 Starkholm A, Kloo L, Svensson PH. Polyiodide hybrid perovskites: a strategy to convert intrinsic $2 \mathrm{D}$ systems into $3 \mathrm{D}$ photovoltaic materials. ACS Appl Energy Mater, 2019, 2: 477-485

9 Herbstein FH, Kaftory M, Kapon M, et al. Structures of three crystals containing approximately-linear chains of triiodide ions. Z für Kristallographie-Crystline Mater, 1981, 154

10 Komsa HP, Senga R, Suenaga K, et al. Structural distortions and charge density waves in iodine chains encapsulated inside carbon nanotubes. Nano Lett, 2017, 17: 3694-3700

11 Lobanov SS, Daly JA, Goncharov AF, et al. Iodine in metal-organic frameworks at high pressure. J Phys Chem A, 2018, 122: 61096117

12 Wlaźlak E, Kalinowska-Tłuścik J, Nitek W, et al. Triiodide organic salts: photoelectrochemistry at the border between insulators and semiconductors. ChemElectroChem, 2018, 5: 3486-3497

13 Mizuno M, Tanaka J, Harada I. Electronic spectra and structures of polyiodide chain complexes. J Phys Chem, 1981, 85: 1789-1794

14 Gao L, Zeng $\mathrm{K}$, Guo J, et al. Passivated single-crystalline $\mathrm{CH}_{3} \mathrm{NH}_{3} \mathrm{PbI}_{3}$ nanowire photodetector with high detectivity and polarization sensitivity. Nano Lett, 2016, 16: 7446-7454

15 Zhou Z, Long M, Pan L, et al. Perpendicular optical reversal of the linear dichroism and polarized photodetection in 2D GeAs. ACS Nano, 2018, 12: 12416-12423

16 Siuzdak K, Szkoda M, Sawczak M, et al. Enhanced photoelectrochemical and photocatalytic performance of iodine-doped titania nanotube arrays. RSC Adv, 2015, 5: 50379-50391

17 Giorgi G, Fujisawa JI, Segawa H, et al. Small photocarrier effective masses featuring ambipolar transport in methylammonium lead iodide perovskite: a density functional analysis. J Phys Chem Lett, 2013, 4: 4213-4216

18 Qiao J, Kong X, Hu ZX, et al. High-mobility transport anisotropy and linear dichroism in few-layer black phosphorus. Nat Commun, 2014, 5: 4475

19 Nour EM, Chen LH, Laane J. Far-infrared and Raman spectroscopic studies of polyiodides. J Phys Chem, 1986, 90: 2841-2846

Acknowledgements This work was financially supported by the National Natural Science Foundation of China (51761145048, 61725401 and 61704097), the Innovation Fund of WNLO and the 62th China Postdoctoral Science Foundation (2017M622418). The authors thank the Analytical and Testing Center of HUST and the facility support from the Center for Nanoscale Characterization and Devices, WNLO. We thank Zhengfeng Guo and Prof. Honggang Gu for optical constant spectra simulation, which are determined by a spectroscopic ellipsometer. We thank Rokas Kondrotas for his kindly help with the English expression of the paper.

Author contributions Yao L and Tang J designed and engineered the experiment and wrote the manuscript; $\mathrm{Xu} \mathrm{P}$ and Chen $\mathrm{S}$ performed the theoretical simulation. Gao W and Gao L contributed to the experiment discussion. Li J and Li D provided the equipment for the polarized Raman measurement. All authors have given approval to the final version of the manuscript.

Conflict of interest The authors declare no conflict of interest.

Supplementary information Supporting data are available in the online version of the paper, including: general XPS spectrum of DMEDA $\mathrm{I}_{6}$ single crystal; partial charge density plots of VBM and CBM; wavelength-dependent responsivity of DMEDA. $\mathrm{I}_{6}$ single crystal photodetection device; polarization absorbance spectrum for 18 um-thick DMEDA $\cdot I_{6}$ single crystal wafer. 


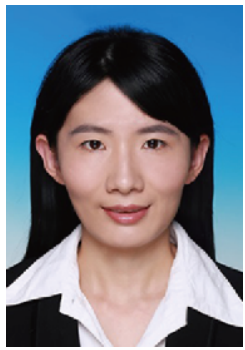

Li Yao received her $\mathrm{PhD}$ degree from the Department of Physics, Peking University in 2016. Now, she is a postdoctor under the supervision of Prof. Jiang Tang in Wuhan National Laboratory for Optoelectronics and School of Optical and Electronic Information, Huazhong University of Science and Technology. Her research interest focuses on the synthesis of organic and inorganic hybrid functional materials and their applications in photoelectronic devices.

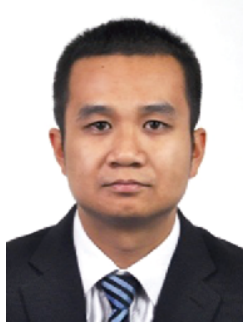

Peng Xu obtained his $\mathrm{PhD}$ degree from Fudan University in 2015 and then joined the Research Institute for Magnetoelectronics \& Weak Magneticfield Detection, College of Science, China Three Gorges University. His research focuses on the calculation of semiconductors for photovoltaic and light emitting applications.

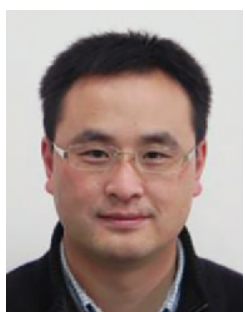

Shiyou Chen is a Professor in the Department of Electronics and the Key Laboratory for Polar Materials and Devices (MOE), East China Normal University. He obtained his $\mathrm{PhD}$ in condensed matter physics from Fudan University in 2009. His research focuses on the calculation study of the multicomponent semiconductors for photovoltaic and photocatalytic applications.

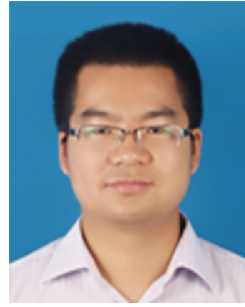

Jiang Tang received his Bachelor's degree from the University of Science and Technology in 2003, and $\mathrm{PhD}$ degree in materials science and engineering from the University of Toronto in 2010. He spent one year and a half as a postdoctoral researcher at IBM T. J. Watson Research Center and then joined Wuhan National Laboratory for Optoelectronics, Huazhong University of Science and Technology as a professor in 2012. His group focuses on antimony selenide $\left(\mathrm{Sb}_{2} \mathrm{Se}_{3}\right)$ thin film solar cells, halide perovskites nanocrystals for light emitting and single crystals for X-ray detection.

\section{通过链状二胺构建强各向异性三碘化合物 DMEDA $\cdot \mathrm{I}_{6}$}

姚利 ${ }^{1}$, 许鹏 ${ }^{2 *}$, 高婉如 ${ }^{1}$, 李俊泽 ${ }^{3}$, 高亮 ${ }^{1}$, 牛广达 ${ }^{1}$, 李德慧 ${ }^{3}$, 陈时有 ${ }^{4,5^{*}}$, 唐江 ${ }^{1,3^{*}}$

摘要 线性链状三碘化物的研究重点主要体现在两个方面: 减小碘 离子之间距离, 有利于电子转移; 提高碘离子排列线性度, 可以实现 强各向异性. 本文采用了一种新策略, 使用链状二胺 $\left(N, N^{\prime}\right.$-二甲基 乙二胺, DMEDA)阳离子与三碘化物离子配位, 形成一维线性三碘 化物DMEDA.I I $_{6}$. 相较于之前报道的三碘化物, 该三碘化物相邻 $\mathrm{I}_{3}{ }^{-}$离子之间的距离最短, 具有较好的线性度; 同时具有 $1.36 \mathrm{eV}$ 的电 子带隙, 具有半导体性能. 理论模拟和实验表征都表明该化合物具 有强的各向异性. 模拟预测沿 $a$ 轴和垂直于 $a$ 轴的吸收差异以及电 子和空穴有效质量的差异最多可达 100 倍. 我们还基于DMEDA $I_{6}$ 构造了一个光电导探测器, 并获得了低暗电流和各向异性的光响 应. DMEDA.I I $_{6}$ 的强各向异性和半导体特性使其成为偏振探测相关 应用的有利竞争者. 